\title{
Should EEG waveform characteristics of BIS may be informative to detect brain fragility and to predict postoperative cognitive dysfunction? A homozygous twins case report
}

\section{Federico Linassi}

Universita degli Studi di Padova Dipartimento di Medicina

\section{Paolo Zanatta}

Universita degli Studi di Verona

Anna Paola Mazzarolo

Azienda ULSS n 2 Marca Trevigiana

Edoardo Rosellini

Università degli studi di Padova

Eleonora Maran ( $\square$ maraneleonora@gmail.com )

Universita degli Studi di Padova Dipartimento di Medicina https://orcid.org/0000-0003-4968-8611

M. Carron

Universita degli Studi di Padova Dipartimento di Medicina

\section{Case report}

Keywords: Intraoperative monitoring, Consciousness monitors, Postoperative cognitive dysfunction

Posted Date: February 27th, 2019

DOI: https://doi.org/10.21203/rs.2.417/v1

License: (c) (i) This work is licensed under a Creative Commons Attribution 4.0 International License.

Read Full License

Version of Record: A version of this preprint was published at Anaesthesia Critical Care \& Pain Medicine on October 1st, 2020. See the published version at https://doi.org/10.1016/j.accpm.2019.12.012. 


\section{Abstract}

Background: Besides inducing behavioral unresponsiveness, a key goal of anesthesia is to prevent the experience of surgery (connected consciousness) by inducing either unconsciousness or disconnection of consciousness from the environment. In pursuing this key goal, avoiding the risk of postoperative cognitive dysfunction is also necessary. In this case report we relate electroencephalography waveform characteristics during intraoperative bispectral index monitoring to long-term neurocognitive decline. Case presentation: We describe the case of two 68-year-old homozygous twins who underwent the same general anaesthesia regimen, but had different results on neurocognitive testing 12 months postoperatively. Neurocognitive tests (Montreal Cognitive Assessment, Trail Making Test A and B, Digit Span Test, and Frontal Assessment Battery) were performed in three different times: the day before surgery, the third day postoperatively, and 12 months after surgery for follow-up. During anaesthesia, connected consciousness and anaesthesia depth were monitored using the isolated forearm technique and bispectral index monitoring. The mean value of bispectral index was lower in one twin (S1) than in the other (S2). Moreover, the same twin who presented lower values of bispectral index had two episodes of burst suppression, whereas the other had none. Regarding the neurocognitive evaluations, both twins did not show significative differences between the tests performed before and after surgery, but an important difference was founded at the 12-month follow-up, when only one twin (S1) showed distinctly worse test scores than previous, denoting a significant cognitive decline. Conclusions: This case-report suggests that bispectral index electroencephalography waveform characteristics during intraoperative bispectral index monitoring may be useful in detecting brain's susceptibility to general anaesthesia and in predicting long-term neurocognitive decline.

\section{Background}

Experience of surgery (defined as connected consciousness 1 ) should be prevented by general anaesthesia, by inducing either unconsciousness or disconnection of consciousness from the environment.

Isolated forearm technique (IFT) and bispectral index (BIS) are two of the most important methods proposed to monitor consciousness during general anaesthesia. Even if BIS values between 40 and 60 are generally recommended as targets for guiding the administration of hypnotics during general anaesthesia2, a recent meta-analysis3 suggest that some patients can exhibit a positive IFT response during surgery with BIS values at range, indicating that to avoid connected consciousness and associated implicit memory (which may lead to adverse psychiatric sequelae, including post-traumatic stress disorder4) may require lowering BIS target values and increasing the depth of anaesthesia.

Regarding this issue, there is concern that increasing the depth of anaesthesia may adversely affect patient outcomes, nevertheless the literature contains conflicting data. For example, deep hypnotic levels have been independently associated with postoperative mortality5-7, but their influence on postoperative cognitive dysfunction (POCD) and delirium has not been established8 9. Furthermore, although previous 
works6-7 in certain at-risk populations suggests that low Mean Arterial Pressure (MAP) and BIS are independently associated with various poor postoperative outcomes, including mortality, Sessler et al found that BIS values < 45 alone, without hypotension and potential cerebral hypoperfusion, was instead associated with a (non-significant) reduction in mortality 10. As far as we know, this is the first study that suggest a possible relation to BIS EEG waveform with the patient brain reserve and his possible neurocognitive outcomes.

\section{Case Presentation}

Two italian 68-year-old male homozygous twins (S1 and S2) who underwent general anaesthesia for total thyroidectomy. They have the same scolarity, lived on different floors of the same apartment with their respective family and did the same job (they were hodmen). Their weight and height were $73 \mathrm{~kg}$ and $174 \mathrm{~cm}$ (S1) and $76 \mathrm{~kg}$ and $175 \mathrm{~cm}$ (S2). Both twins had essential hypertension that was well controlled with beta-blockers and, prior to this, neither of them has undergone surgery.

Each twin received an identical general anaesthesia regimen. No premedication was administered. Anaesthesia was induced with fentanyl $150 \mathrm{mcg}$ and propofol $2 \mathrm{mg} \mathrm{kg}-1$, and neuromuscular blockade (NMB) was achieved with rocuronium $1 \mathrm{mg} \mathrm{kg}-1$. After orotracheal intubation, anaesthesia was maintained with desflurane at a minimum alveolar concentration of approximately 0.8 (5.4\% vaporizer setting on the Dräger Zeus ${ }^{\circledR}$ Infinity ${ }^{\circledR}$ Empowered anaesthesia machine). Two fentanyl boluses were administered: 150 mcg just before skin incision and $100 \mathrm{mcg} 40$ minutes later. Rocuronium $0.15 \mathrm{mg} \mathrm{kg}-1$ boluses were administered to maintain a deep NMB (absence of first twitch at train-of-four stimulation). Thirty minutes before the end of surgery, the patients received paracetamol $1000 \mathrm{mg}$, ketoprofen $160 \mathrm{mg}$, and ondansetron $4 \mathrm{mg}$ for pain, nausea and vomiting prophylaxis, respectively. At the conclusion of surgery, deep NMB was reversed with sugammadex $4 \mathrm{mg} \mathrm{kg-1}$ and the desflurane was, then, discontinued.

Both twins underwent BIS, IFT, and train-of-four (TOF) monitoring throughout anaesthesia. BIS and TOF monitors were placed before anaesthesia induction and removed after emergence. The tourniquet used for IFT monitoring was placed on the dominant arm before induction. It was inflated after injection of propofol and fentanyl, but before the patients received any rocuronium. The tourniquet was deflated only after complete reversal of NMB (verified by TOF) at the end of surgery. IFT testing was performed at 5minute intervals throughout surgery. The patients wore headphones through which they heard a recorded voice saying, "Name, name, it's your anaesthesiologist speaking. If you can hear me, squeeze your hand... [10 seconds pause] ... Name, name, it's your anaesthesiologist speaking. If you feel pain, squeeze your hand twice" (with "name" replaced by the name of the patient).

Both twins underwent the same neuropsychological tests at three different times: the day before surgery (T0), 3 days after surgery (T1), and 12 months after surgery (follow-up). Montreal Cognitive Assessment (MoCA), Trail Making Test A (TMT-A) and B (TMT-B), Forward and Backward Digit Span Tests (DST), and Frontal Assessment Battery (FAB) were performed. These tests assessed cognitive function, including 
short-term memory, working memory, visuo-spatial research ability, selective and divided attention, psychomotor speed and executive functions. The test results were collected, corrected for age and level of schooling, and then interpreted by a psychologist (APM). Both cut-off values (26/30 for MoCA, 94 seconds for TMT-A, 283 seconds for TMT-B, 3.75/9 for DST Forward, and 3/9 for DST Backward) and zscores (for FAB) were considered when evaluating mental impairment. Cognitive deficit was defined as a score below the cut-off value for the MoCA and DST tests, a score above the cut-off for the TMT tests, or a $z$-score of at least -1.0 for the FAB test ( 1 or more standard deviation reduction in FAB performance).

\section{$\underline{\text { Results }}$}

Neither twin required vasoconstrictor drugs during general anaesthesia, as their intraoperative haemodynamic parameters were stable. Neither twin reported explicit recall.

\section{Neurological monitoring results}

Before induction of anaesthesia the BIS values for S1 and S2 were 97 and 98, respectively. In both patients, the mean BIS was $<60$ during anaesthesia. The mean bis was 35 for S1 and 42 for S2. Furthermore, 2 episodes of burst suppression were observed on the BIS monitor's electroencephalography (EEG) waveform display for S1: the first occurred after skin incision and lasted 12 minutes, and the second occurred approximately 40 minutes after skin incision (after the $100 \mathrm{mcg}$ fentanyl bolus) and lasted 8 minutes. No burst suppression was observed for S2. Intraoperative BIS values are summarized in Table 1.

Neither patient exhibited a positive IFT response during surgery. S1 had a positive response 8 minutes after discontinuing desflurane and was extubated 3 minutes later. He was confused (referring to hospital personnel as his mother) for approximately 10 minutes after awakening. $S 2$ had a positive IFT response 6 minutes after discontinuing desflurane and was extubated 4 minutes later. He was confused (thought he was at park) for approximately 5 minutes after awakening. Neither patient exhibited arm complications related to ischemia induced by the tourniquet.

IFT response during surgery are shown in Table 1.

\section{Neurocognitive testing results}

Preoperatively, the scores obtained at MoCA, FAB and Backward DST tests fulfilled the definition of cognitive deficit for both twins. At 3 days postoperatively, the scores on all tests worsened in both twins, except for the Forward and Backward DST scores which remained the same as preoperatively. For both the preoperative and postoperative tests, the results for $\$ 1$ were approximately the same as those for $\mathrm{S} 2$. At the 12-month follow-up, the neurocognitive test results for $\mathrm{S} 2$ were generally similar to his postoperative results; by contrast, $\mathrm{S} 1$ had clear worsening of all test results, with all values fulfilling (or almost fulfilling [TMT-A score was at the cut-off value]) the definition of cognitive deficit.

Neurocognitive test results are shown in Table 1. 
Table 1: Neurocognitive test results and intraoperative monitoring values.

BIS: bispectral index; DST: Digit Span Test; FAB: Frontal Assessment Battery; IFT: isolated forearm technique; MoCA: Montreal Cognitive Assessment; TMT: Trail Making Test. Neurocognitive results in parentheses (c) represent age- and schooling-corrected values.

\section{Discussion And Conclusions}

This case report focused on two important anaesthesia themes: connected consciousness during general anaesthesia and POCD after anaesthesia delivery.

Neither of our patients exhibited a positive IFT response, suggesting that they did not experience connected consciousness. However, an IFT response is quite frequent during general anaesthesia (34.8\%), especially during maintenance (31.2\%), using intravenous anaesthesia (48\%), and targeted BIS values of 45 to $60(57 \%) 3$. The risk of connected consciousness can be reduced by achieving a deeper level of anaesthesia. In this regard, there is concern that deep anaesthesia may increase the risk of POCD, but this issue remains controversial. In their large randomized trial, Chan et al. 11 found that BIS-guided anaesthesia with a target of 40 to 60 reduced the incidence of POCD from $14.7 \%$ to $10.2 \%$ three months postoperatively, compared with routine anaesthesia management. By contrast, Farag et al.12 reported less POCD in patients with a greater depth of isoflurane anaesthesia, and An et al.13 found that patients with lower BIS values during most of their procedure had a lower incidence of early POCD. In a prospective observational study, Soehle et al.14 concluded that the burst suppression ratio may identify patients at risk for POCD following cardiac surgery, although it is unknown whether the association between burst suppression and POCD represents a causal relationship.

The two patients described in this report received the same anaesthesia regimen and had similar perioperative neurocognitive test results. Nevertheless, S1 exhibited substantial worsening of neurocognitive tests one year after surgery, with short-term memory, working memory, and frontal functions (conceptualization, mental flexibility, motor programming, sensitivity to interference, inhibitory control, environmental autonomy) being most affected. Although the cognitive decline of S1 was not predicted by any of the post-operative tests, which indeed showed no differences between the twins, BIS values during anaesthesia differed between the two patients. Specifically, the mean BIS value was lower in $\mathrm{S} 1$ than in S2, and burst suppression occurred only in S1. These results should indicate that the brain of $S 1$ was more sensitive to anaesthesia than that of S2, suggesting that S1 had greater brain fragility and less brain reserve.

In conclusion, with this case report we would suggest that BIS values and EEG waveform characteristics during intraoperative BIS monitoring may be useful for predicting brain susceptibility to general anaesthesia and neurocognitive decline in the long term, even with inalterated neuropsychological test scores at T1. 
The major limit of this case report is the absence of further neuropsychological follow-up evaluations that could also be done closer to surgery (like also 3-6 months later and not just 12 months later).

However, 18 months after surgery (the moment we write), whereas S2 was still living at his home, S1 was recovered in a long-stay hospital for elderly suggesting that, despite this limit, neurocognitive decline is confirmed and still present, if not worsened, in time.

Future studies could investigate the correlation between BIS values and EEG waveform characteristics and the brain fragility of the patients and the onset of POCD, and if peri-operative strategies can reduce the incidence of POCD, specially in the elderly.

\section{List Of Abbrevations}

IFT: Isolated forearm technique

BIS: Bispectral Index

POCD: Postoperative cognitive dysfunction

MAP: Mean arterial pressure

NMB: neuromuscular blockade

TOF: train-of-four

MoCA: Montreal Cognitive Assessment

TMT: Trail Making Test

DST: Digit Span Test

FAB: Frontal Assessment Battery

\section{Declarations:}

\section{Ethical approval and consent to partecipate}

Both patients (S1 and S2) provided written informed consent for participation in this case report. Ethical approval was not applicable since monitoring and treatments of patients involved do not differ form routinely clinical practice of our hospital.

\section{Consent to publish}

Written informed consent was obtained from the participants for publication of this article and any accompanying tables/images. A copy of the written consent is available for review by the Editor of this journal. 
This case report contains clinical data from medical records in our hospital. The datasets used in the current case are available from the corresponding author upon reasonable request.

\section{Competing interests}

There are no competing interests.

\section{Funding}

No financial support was received for this work.

\section{Author's contributions:}

FL and PZ conceived of the study; acquired, collected, and analysed data; and drafted and revised the final manuscript. APM, EM and ER collected and analysed data, and revised the final manuscript. MC participated in conceiving the study, analysing the data, discussing the results, and revising the final manuscript. All authors read and approved the manuscript.

\section{Acknowledgements}

The authors would like to thank Alessandro De Cassai, MD, of the Department of Medicine, Anaesthesiology and Intensive Care, University of Padova, for his contribution in data collecting.

\section{Bibliography}

Sanders RD, Tononi G, Laureys S, Sleigh JW. Unresponsiveness $\neq$ unconsciousness. Anesthesiology 2012; 116: 946-59

Sebel PS, Lang E, Rampil IJ, et al. A multicenter study of bispectral electroencephalogram analysis for monitoring anesthetic effect. Anesth Analg 1997; 4: 891-9

Linassi F, Zanatta P, Tellaroli P, Ori C, Carron M. Isolated forearm technique: a meta-analysis of connected consciousness during different general anaesthesia regimens. Br J Anesth 2018; 121: 198-209.

Osterman JE, Hopper J, Heran WJ, Keane TM, van der Kolk BA. Awareness under anesthesia and the development of posttraumatic stress disorder. Gen Hosp Psychiatry 2001; 23: 198-204

Monk TG, Saini V, Weldon BC, Sigl JC. Anesthetic management and one-year mortality after noncardiac surgery. Anesth Analg 2005; 100:4-10

Kertai MD, Pal N, Palanca BJ et al. Association of perioperative risk factors and cumulative duration of low bispectral index with intermediate-term mortality after cardiac surgery in the B-Unaware Trial. Anesthesiology 2010; 112: 1116-27 
Leslie K, Myles PS, Forbes A, Chan MT. The effect of bispectral index monitoring on long-term survival in the B-aware trial. Anesth Analg 2010; 110:816-22

Steinmetz J, Funder KS, Dahl BT, Rasmussen LS. Depth of anaesthesia and post-operative cognitive dysfunction. Acta Anaesthesiol Scand 2010; 54:162-8

Hou R, Wang H, Chen L, Qiu Y, Li S. POCD in patients receiving total knee replacement under deep vs light anesthesia: A randomized controlled trial. Brain Behav 2018; 30: e00910

Sessler DI, Sigl JC, Kelley SD, et al. Hospital stay and mortality are increased in patients having a "triple low" of low blood pressure, low bispectral index, and low minimum alveolar concentration of volatile anesthesia. Anesthesiology 2012; 116: 1195-203.

Chan MT, Cheng BC, Lee TM, Gin T; CODA Trial Group. BIS-guided Anesthesia Decreases Postoperative Delirium and Cognitive Decline. J Neurosurg Anesthesio/ 2013; 25: 33-42

Farag E, Chelune GJ, Schubert A, et al. Is depth of anesthesia, asassessed by the bispectral index, related to postoperative cognitive dysfunction and recovery? Anesth Analg 2006; 103: 633-640.

An J, Fang Q, Huang C, Qian X, et al. Deeper Total Intravenous Anesthesia Reduced the Incidence of Early Postoperative Cognitive Dysfunction After Microvascular Decompression for Facial Spasm. J Neurosurg Anesthesiol 2011; 23: 12-7

Soehle M, Dittmann A, Ellerkmann RK, Baumgarten G, Putensen C, Guenther U. Intraoperative burst suppression is associated with postoperative delirium following cardiac surgery: a prospective, observational study. BMC anesthesiol 2015; 28: 15-61

\section{Tables}

Table 1: Neurocognitive test results and intraoperative monitoring values.

BIS: bispectral index; DST: Digit Span Test; FAB: Frontal Assessment Battery; IFT: isolated forearm technique; MoCA: Montreal Cognitive Assessment; TMT: Trail Making Test. Neurocognitive results in parentheses (c) represent age- and schooling-corrected values.

Due to technical limitations the table for this paper has been uploaded as a supplemental file.

\section{Supplementary Files}

This is a list of supplementary files associated with this preprint. Click to download.

- supplement1.pdf

- supplement2.jpg 